\title{
La ilegalidad del muro de Donald Trump
}

\author{
The illegality of Donald Trump's wall
}

\section{Illegalite du mur de Donald Trump}

\section{Manuel Becerra Ramírez*}

SUMARIO: I. Introducción. II. Los antecedentes del muro. III. La Orden Ejecutiva del presidente Trump. IV. Impacto ambiental del muro. V. ¿Un muro fronterizo es ejercicio legal de la soberanía estatal? VI. Marco jurídico internacional. VII. Tratados México-Estados Unidos. VIII. Los recursos transfronterizos. IX. El derecho convencional y el principio de no perjudicar al medio ambiente de otro Estado, con actividades dentro de su territorio. X. Conclusiones. XI Bibliografía.

* Investigador del Instituto de Investigaciones Jurídicas de la UNAM. Investigador emérito del Sistema Nacional de Investigadores.

Artículo recibido el 6 de julio de 2019

Aprobado para publicación el 3 de octubre de 2019 
RESUMEN: En este trabajo se analiza, desde la perspectiva del derecho internacional, el impacto de la construcción de un muro por parte del gobierno de los Estados Unidos en la frontera con México. Con base en estudios de expertos en ecología se analiza el impacto en el ecosistema la introducción de un elemento agresivo, intrusivo como la construcción de un muro de la región fronteriza. La frontera está regulada por una gran cantidad de normas jurídicas internacionales, las cuales, con la construcción de un muro, Estados Unidos viola al dañar, en forma irreversible, el ecosistema de la región. Precisamente el derecho internacional contemporáneo en materia de medio ambiente limita la soberanía interna de los Estados, en relación con actos que puedan dañar a los vecinos.

Palabras clave: fronteras y derecho internacional, derecho internacional del medio ambiente, muros fronterizos en derecho internacional, principios del derecho de medio ambiente, frontera México-Estados Unidos.

ABSTRACT: This paper analyzes, from the perspective of international law, the impact of the construction of a wall by the United States government on the border with Mexico. Based on studies of experts in ecology, the impact on the ecosystem of the introduction of an aggressive and intrusive element such as the construction of a wall in the border region is analyzed. The border is regulated by a large number of international legal norms, which, with the construct nion of a wall, the United States violates by irreversibly damaging the ecosystem of the region. Precisely, contemporary international environmental law limits the internal sovereignty of States, in relation to acts that may harm neighbours.

Key words: Borders and International Law, International Environmental Law, Border Walls in International Law, Principles of Environmental Law. Mexico-United States Border.

RÉSUMÉ: Cet article analyse, du point de vue du droit international, l'impact de la construction d'un mur par le gouvernement des États-Unis à la frontière avec le Mexique. Sur la base d'études menées par des experts en écologie, nous analysons l'impact sur l'écosystème de l'introduction d'un élément agressif et intrusif, tel que la construction d'un mur dans la frontière. La frontière est réglementée par un grand nombre de normes juridiques internationales qui, avec la construction d'un mur, sont violées par les États-Unis en endommageant de manière irréversible l'écosystème de la région. C'est précisément le droit international de l'environnement contemporain qui limite la souveraineté interne des États en ce qui concerne les actes susceptibles de nuire aux voisins.

Mots-clés: frontières et droit international, droit international de l'environnement, barrières frontalières en droit international, principes du droit de l'environnement, frontière Mexique-États-Unis. 


\section{INTRODUCGIÓN}

Es cierto que parte del ejercicio de la soberanía estatal consiste en el control de las fronteras y la determinación de quién entra y quién no al territorio de los Estados. Y también es cierto que la historia de la humanidad está llena de muros. La experiencia histórica nos dice que, esos muros ahora son monumentos arquitectónicos impresionantes, construidos con motivos de defensa, al mismo tiempo son símbolos oprobiosos (el muro de Berlín, por ejemplo) o manifestación de masivas violaciones de derechos humanos, como son los casos de la muralla China, en donde las crónicas dicen que para su construcción murieron aproximadamente 10 millones de trabajadores o bien el muro construido por Israel, en el territorio en disputa con Palestina, y que la Corte Internacional de Justicia ha declarado violatorio del derecho internacional.

Aún más, el actual muro construido en la frontera México-Estados Unidos tiene una intención malévola, al dejar abierto el espacio desértico no impide la entrada a los Estados Unidos, sino que la hace más peligrosa, difícil y en muchísimos casos mortal. Evidentemente hay una violación flagrante a los derechos humanos de miles de personas que no son delincuentes, sino que buscan una forma digna de vivir.

Ya desde la campaña hacia la presidencia, Donald Trump ofreció la construcción de un muro en la frontera con México, y que los mexicanos, por alguna vía lo iban a pagar; así, unos cuantos días después de tomar la presidencia, Donald Trump, emitió una serie de órdenes ejecutivas de las cuales la primera será objeto de nuestros comentarios, en la que cumple con su amenaza, al ordenar la construcción de un muro. Este acto de Estado tiene una gran trascendencia para el orden jurídico internacional y concretamente para la relación bilateral México-Estados Unidos, pues independientemente de que viola el orden jurídico internacional significa un acto inamistoso en contra de sus vecinos sureños.

Desde que México adquiere su independencia en el siglo XIX, la frontera entre los dos países se ha movido impresionantemente desfavoreciendo a este país. Actualmente, es una de las fronteras más largas del mundo $(3,141 \mathrm{~km}$ de longitud) en la que colindan cuatro Estados estadounidenses (California, Arizona, Nuevo México y Texas) y, seis Estados mexicanos 
Esta revista forma parte del acervo de la Biblioteca Jurídica Virtual del Instituto de Investigaciones Jurídicas de la UNAM

(Baja California Norte (BCN), Sonora, Chihuahua, Coahuila, Nuevo León y Tamaulipas) $;^{1}$ y además es muy rica, pues a pesar de parecer, y ser, árida, desértica, la región fronteriza está llena de vida silvestre, mucha de la cual es endémica y está en peligro de extinción.

En este trabajo, en el marco del derecho internacional contemporáneo, analizamos el impacto de la política de los Estados Unidos en la frontera, concretamente con la construcción de un muro que ya está parcialmente colocado en la región y lo que significa su prolongación.

El presente trabajo se desarrolló durante mi estancia sabática en la Universidad British Columbia, de Vancouver, Canadá (septiembre 2018-agosto 2019) con el soporte del Programa de Apoyos para la Superación del Personal Académico de la UNAM (PASPA) de la Dirección General del Personal Académico (DGAPA), a los cuales agradezco profundamente por su sustento.

\section{LOS ANTECEDENTES DEL MURO EsTADOS UNIDOS-MÉXICO}

La historia de la idea de construcción del muro en la frontera se remonta a la década de los 80, cuando la afluencia de migrantes mexicanos hace reaccionar al gobierno de los Estados Unidos con “muros” legales. En 1986 se dictó el Immigration Reform and Control Act, que sancionaba a quien, sabiéndolo, contratara a un inmigrante ilegal.

Después, en 1990 la U.S. Border Patrol empezó a construir barreras físicas en el sector de San Diego, colocándose 14 mil millas de vallas entre San Diego y Tijuana. Junto con ello, se aprobó la Immigration Act que pretendía beneficiar a los migrantes pues aumentó el número de visas para los trabajadores y profesionales.

En 1993 se dicta la Operation Blockade en El Paso, Texas y al año siguiente, 1994, la Operation Gatekeeper en San Diego, California. En virtud de estas operaciones se construyó un muro en la parte que hay más movimiento de personas en tránsito lo que hizo correr el flujo de trabajadores migrantes hacia el desierto de Sonora y Arizona de parte del territorio estadounidense. Este movimiento trajo varias consecuencias.

Anglés Hernández, Marisol, "Los cursos de agua compartidos entre México y los Estados Unidos de América y la variable medio ambiental. Una aproximación”, Anuario Mexicano de Derecho Internacional, México, vol. VI, 2006, pp. 89-166. 
En principio, aumentaron las muertes de los mexicanos que quisieron cruzar la frontera; después aumentó el costo a pagar a los traficantes (polleros) de trabajadores migrantes. Por último, se produjo un efecto que no habían previsto los estadounidenses: los mexicanos, en lugar de regresar a sus lugares de residencia, prefirieron quedarse en territorio extranjero, y aún más, cuando no pudieron regresar a su país, les siguieron sus familias y esto hizo que se incrementara el número de niños de migrantes nacidos en Estados Unidos. Esta política anti inmigrante se agudizó con la reforma de 1996 denominada Illegal Immigration Reform and Immigrant Responsibility Act of 1996 bajo la presidencia del presidente W. Clinton, que penalizó la migración, agravando, por supuesto, la situación legal de los migrantes mexicanos. ${ }^{2}$

Sin embargo, el gobierno de Estados Unidos siguió insistiendo con su muro. En 2006, el presidente de los Estados Unidos, George W. Bush, firmó la ley denominada Secure Fence Act, mediante la cual se le autoriza la construcción de una valla fronteriza entre México y Estados Unidos cerca de El Paso, Texas, una extensión de 700 millas adicionales $(1,100 \mathrm{~km})$ "de doble cadena y cercas de alambre de púas con luz y postes de cámara infrarrojos”. Además, autorizó al Department of Homeland Security para incrementar el uso de tecnología avanzada como cámaras, satélites y vehículos aéreos para reforzar la infraestructura de la frontera. La idea de esta ley fue hacer más segura la frontera del sur de los Estados Unidos y en aquel tiempo, el presidente Bush, la consideraba un paso importante hacia una reforma migratoria. ${ }^{3}$

De 2006 a 2017 se han seguido construyendo vallas a lo largo de la frontera y no sólo eso, sino que, se han construido una segunda y tercera valla,

2 Además, hay que dejar solamente anotado, pues no es el objeto de este trabajo, supuestamente uno de los objetivos delTLCAN era crear empleos en el país que detuvieran la migración hacia los Estados Unidos. Sin embargo, no solamente no se detuvo el flujo de los mexicanos, sino que algunos deciden emigrar, a pesar de los enormes peligros, incluso de muerte, que eso implica a través del desierto. Las razones pueden ser la falta de empleo, pero también la gran diferencia que hay entre los sueldos mexicanos y los estadounidenses. Eso significa que el mencionado tratado de libre comercio no tuvo el efecto que tanto se publicitó. También la frontera siguió tan porosa con y sin muros, pues la droga sigue fluyendo y también el dinero del narcotráfico y las armas. Esto debería ser tomado en cuenta en las negociaciones con el vecino del norte, pues es ilógico pensar que, con tanto gasto en seguridad en la frontera, los narcotraficantes sigan alcanzando su objetivo de surtir la enorme demanda de drogas del lado estadounidense. Independientemente del exitoso negocio de venta de armas en el Estado mexicano.

3 Secure Fence Act of 2006, disponible en: https://ballotpedia.org/Secure_Fence_Act_ of_2006, consultada el 4 de octubre de 2019. 
lo que además del gasto por reparaciones ha significado un considerable gasto para el presupuesto estadounidense. Según reportes de 2017, se informó que desde el año fiscal de 2007 al 2016, se gastaron cerca de 2,300 millones en la construcción de la valla a lo largo de la frontera sud oriental y además que se pronosticó que se requerirán sumas considerables para su mantenimiento (mil millones en los próximos 20 años). ${ }^{4}$ Esta política migratoria de vallas selectivas ha provocado muchas muertes de trabajadores migrantes por el cruce del desierto. ${ }^{5}$

Por otra parte, el flujo migratorio de mexicanos a los Estados Unidos, según los reportes de los expertos, llegó a su máxima expresión en el año de 2007, y después empezó a bajar en virtud de que "se terminó el bono demográfico, se prefiere la migración legal, ya no sale a cuenta ir al norte dado el incremento de costos, riesgos y penas", ${ }^{6}$ pero no sucedió lo mismo con los migrantes centro americanos, que aumentaron su flujo.

También hay que mencionar que no obstante la construcción de obstáculos para el paso a territorio norteño, ello no ha impedido que los traficantes de drogas puedan pasar por ahí, pues han utilizado cuerdas para escalar la valla, rampas para poder pasar vehículos, han construido túneles, cortando la valla, y aún utilizados aviones pequeños para sortear el paso; lo que si ha logrado es fragmentar el ecosistema, afectando a las especies vivas que lo habitan, como ya lo veremos más adelante.

\section{LA ORDEN EJECUTIVA DEL PRESIDENTE TRUMP}

El 25 de enero del 2017, con sólo pocos días de haber tomado el poder, el presidente de los Estados Unidos Donald Trump emitió una orden ejecutiva (Executive Order), que en el sistema legal mexicano se equipararía a un reglamento; es decir, que es una facultad del presidente estadounidense emitirla. La orden ejecutiva se basa en la preocupación por la pérdida del control de la frontera sur, y lo toma como un elemento de seguridad nacional (Border

4 Idem.

5 Ya desde la aprobación de la Secure Fence Act de 2006, los oponentes a la ley preveían que la valla no detendría a los migrantes, sino que los empujarían a tomar "rutas traicioneras" (treacherous routes). Idem.

6 Durand, Jorge, "Entre la línea y el muro", La Jornada, disponible en: https: / /www.jornada.com.mx/2019/01/20/opinion/020a2pol. Consultada el 20 de enero de 2019. 
security is critically important to the national security of the United States). Lo cual podría considerarse un elemento discursivo, pero como sabemos, de los antecedentes de la política exterior de la gran potencia estadounidense, cuando algo es considerado parte de la seguridad nacional se justifica la utilización de la fuerza, como una potencia imperial.

El análisis de la orden ejecutiva puede ser realizado desde dos perspectivas: desde el derecho interno estadounidense, y desde la perspectiva del derecho internacional, al cual nos avocaremos en este trabajo.

En la orden ejecutiva, el gobierno de Estados Unidos pone el acento en la "emigración ilegal", y el crimen organizado y establece los siguientes puntos de la política del gobierno estadounidense:

Sec. 2. Es política de la rama del ejecutivo:

(a) Asegurar la frontera sur de los Estados Unidos a través de la construcción inmediata de un muro físico en la frontera sur, vigilado y apoyado por el personal adecuado a fin de prevenir la emigración ilegal, el tráfico de drogas y humanos y los actos de terrorismo;

(b) Detener a las personas que hayan sido arrestadas por sospecha de violar las leyes federales o estatales, incluida la ley federal de inmigración, y que se encuentren pendientes de nuevos procedimientos relativos a esas violaciones;

(c) Agilizar las decisiones de las reclamaciones, de los individuos detenidos, de ser elegibles para permanecer en los Estados Unidos;

(d) Retirar con prontitud a aquellas personas cuyas reclamaciones legales para permanecer en los Estados Unidos hayan sido rechazadas legalmente, después de que se hayan impuesto sanciones civiles o penales apropiadas y;

(e) cooperar plenamente en la aplicación de las leyes estatales y locales en la promulgación de las asociaciones Federal-Estatal para hacer cumplir las prioridades federales de inmigración, así como los programas estatales de vigilancia y detención que son consistentes con la ley federal y no socavan las prioridades federales de inmigración. ${ }^{7}$

7 Traducción propia. Sec. 2. Policy. It is the policy of the executive branch to:

(a) secure the southern border of the United States through the immediate construction of a physical wall on the southern border, monitored and supported by adequate personnel to prevent illegal immigration, drug and human trafficking, and acts of terrorism;

(b) detain individuals apprehended on suspicion of violating Federal or State law, including Federal immigration law, pending further proceedings regarding those violations; 
Esta disposición ejecutiva del gobierno estadounidense, que se refiere también a la construcción de un muro en la frontera sur de los Estados Unidos, es decir, en la frontera con México, entraña una posible violación de los derechos de los mexicanos en el territorio de los Estados Unidos.

La orden ejecutiva es un acto de Estado que al trascender las fronteras estatales está sujeto al escrutinio, a través del derecho internacional. En principio, la construcción de un muro tiene un impacto impresionante en el ecosistema que no reconoce fronteras entre Estados Unidos y México y en consecuencia una violación a todo un tejido de normas de derecho convencional, bilateral y multilateral, consuetudinario, inclusive de normas de lege ferenda. Como nos disponemos a analizar a continuación.

\section{IMPACTO AMBIENTAL DEL MURO}

El muro como las vallas construidas en la frontera, así como, los posibles muros que se puedan construir ya han sido estudiados por los científicos de ambos lados de la frontera. Al respecto destaca el estudio de los científicos Jesse R. Lasky, Walter Jetz y Timothy H. Keitt, que desde hace una década advertían sobre los daños al ecosistema con la fragmentación de la zona geográfica fronteriza:

Principales conclusiones: Las nuevas barreras a lo largo de la frontera aumentarían el número de especies en riesgo, especialmente en las tres regiones identificadas, lo que debería priorizarse para mitigar los impactos de las barreras actuales. Las es-

(c) expedite determinations of apprehended individuals' claims of eligibility to remain in the United States;

(d) remove promptly those individuals whose legal claims to remain in the United States have been lawfully rejected, after any appropriate civil or criminal sanctions have been imposed; and

(e) cooperate fully with States and local law enforcement in enacting Federal-State partnerships to enforce Federal immigration priorities, as well as State monitoring and detention programs that are consistent with Federal law and do not undermine Federal immigration priorities. 
DOI: http://dx_doi_org/10.22201/iij_24487872e_2020_20.14495

pecies que identificamos como potencialmente en riesgo merecen un estudio más profundo para determinar los impactos de las barreras de dispersión fronteriza. ${ }^{8}$

Hay que decir también, que la preocupación sobre la conservación de la flora y fauna de la frontera, México-Estados Unidos, no solo es binacional, sino que igualmente es una preocupación trinacional, si lo vemos con el Acuerdo celebrado entre Canadá, los Estados Unidos y México para la conservación y administración del ecosistema y la vida silvestre y mediante el cual se crea un Comité Trilateral. ${ }^{9}$

También del lado mexicano, los expertos en medio ambiente se han referido puntualmente a las consecuencias para el entorno natural que puede tener la construcción de un muro en la frontera; pero, es más, ya es posible medirlas en la parte que está construida, de tal manera que no se habla de hipótesis, sino de realidades. ${ }^{10}$

La geografía de América del norte nos muestra regiones que forman ecosistemas intensamente integrados, como los desiertos de Chihuahua y Sonora, que se extienden a lo largo del territorio norteño de México y parte del territorio de Estados Unidos, que al mismo tiempo que son ricos en vida animal y vegetal se encuentran en un equilibrio biológico débil que requieren un cuidado y protección de la acción del ser humano. ${ }^{11}$

En efecto, en la frontera hay una gran cantidad de especies vegetales y animales que, inclusive, no tienen una limitación geográfica como la que

8 Lasky, Jesse R. et al., "Conservation biogeography of the US-Mexico border: a transcontinental risk assessment of barriers to animal dispersal", Diversity and Distributions. A Journal of Conservation Biogeography, First published: 3 May 2011, disponible en: https: / doi. org/10.1111/j.1472-4642.2011.00765.x, consultado el 10 de marzo de 2019, trad. de MBR.

9 Memorandum of Understanding establishing the Canada/Mexico/US Trilateral Committee for Wildlife and Ecosystem Conservation and Management (Trilateral Committee) https: / / www.fws.gov/international/wildlife-without-borders/mexico/trilateral-committee.html, Consultado el 17 de enero del 2019.

10 Por ejemplo, en el año de 2011, en parte debido a fuertes lluvias se produjo una inundación en el punto fronterizo de Nogales Sonora, la causa principal fue el semibloqueo de un desagüe que cruza la frontera. Véase: "Reserva de la Biosfera el Pinacate y Gran Desierto del Altar, Reservas del Programa "El Hombre y la Biosfera (MAB)" de la UNESCO, desde el 25 de octubre de 1995”, disponible en: http://sil.gobernacion.gob.mx/Archivos/Documentos/2017/09/asun_357 3146_20170914_1505400463.pdf (consultado el 22 de diciembre del 2018).

11 Véase Hernández, Héctor, M., La vida en los desiertos mexicanos, México, Fondo de Cultura Económica, 2006. 
establecen las fronteras estatales, pues son trashumantes, o mejor dicho en términos jurídicos, son transfronterizos, como es el caso del león de la montaña y el gato montés, las aves, los osos negros, berrendos, jaguares, lobos mexicanos, ocelotes que, insistimos, no reconocen limites políticos creados por el ser humano. Algunas de ellas sufrirían, con el muro, un impacto inmediato y evidente como son los casos del correcaminos y el búho pigmeo que vuela a bajas alturas.

Simplemente, en la frontera con el estado de Sonora se localizan el jaguar, el borrego cimarrón y los berrendos, venados, gatos salvajes, coyotes y lobos que, se mueven libremente en la frontera y los cuales ya tienen una reserva especial, protegida por los dos países. ${ }^{12}$ En efecto, este ecosistema fronterizo que abarca el Refugio Nacional de Vida Silvestre (NationalWildlife Refuge) Cabeza Prieta en Arizona y el desierto de Pinacate y Gran Altar en el Estado mexicano de Sonora, está protegido por los dos países y certificado como patrimonio de la humanidad por la Organización de las Naciones Unidas para la Educación, la Ciencia y la Cultura (UNESCO). Para marcar los límites entre los dos países existe una cerca, precisamente diseñada para el tránsito de los animales de la vida silvestre.

El libre tránsito de los animales en esta región es vital, porque ellos se mueven en busca de agua, alimentos, e inclusive de parejas, como es el caso de los jaguares que solamente existen cinco ejemplares en el territorio de los Estados Unidos y tienen que pasar al territorio mexicano para encontrar pareja. Eso sucede también con el medio ambiente natural que se retroalimenta de la acción de los animales ya que impacta la flora especial de esta región (por ejemplo, los animales esparcen las semillas de las plantas). Es todo un ecosistema que tiene su armonía y que si se rompe con un muro (aunque sea "bonito", como dice Trump) tendría un impacto indudable en todo el ecosistema.

El caso del berrendo es importante. Esta es una especie, que se encuentra en el territorio de Sonora y Arizona y está a punto de extinción por lo que la colaboración de los dos Estados es necesaria para evitarla. La mayor parte de los berrendos se encuentra en territorio mexicano, en el Estado de Sonora; sin embargo, los dos gobiernos realizan una colaboración, que

12 Ortega, Yemeli, “Trump wall threatens Mexico's animals without borders”, April 9, 2017, disponible en: https://phys.org/news/2017-04-trump-wall-threatens-mexico-animals. html\#jCp. Consultado el 17 de enero del 2019. 
es ejemplar, al nivel de funcionarios del medio ambiente, con la finalidad de que se mantenga la especie; ${ }^{13}$ lo mismo sucede con organismos no gubernamentales, como la Agrupación Sierra Madre, S.C, y el Departamento de Caza y Pesca de Nuevo México. ${ }^{14}$

Aún, con la carretera y la cerca construida, en la frontera hay datos que muestran que, sí hay una comunicación entre la población de berrendos. "Sin embargo, los planes de construir una muralla acabarán entonces con estas migraciones ocasionales, afectando el intercambio de material genético necesario para la supervivencia a largo plazo del berrendo". ${ }^{15}$

El lobo mexicano, al igual que el berrendo, se encuentra en el territorio norteño de los Estados Unido y México y es asimismo una especie que está en peligro de extinción, por esta razón, los Estados Unidos en el año de 1970 la declararon en este estatus. En este caso también hay una estrecha colaboración binacional para que se reproduzca. Precisamente, es necesario que haya espacio libre para que los lobos puedan migrar y comunicarse con otros de su especie del otro lado de la frontera. Del mismo modo, aquí la construcción de un muro impenetrable pone en peligro a la especie. ${ }^{16}$

Otro animal amenazado por la acción del hombre es el bisonte que, después de la existencia de millones en las praderas de lo que ahora es América del norte, actualmente necesita de la cooperación internacional para sobrevivir. Como una prueba de que es necesario tener una frontera "porosa", por razones del movimiento de los animales, hay que mencionar que los bisontes tienen el pasto en el territorio de los Estados Unidos, pero el agua en México, por eso se mueven y rompen las vallas que ahora existen, lo que

13 Las normas mexicanas y estadounidenses prevén la conservación de los berrendos; así, tanto la NOM-059-SEMARNAT, como la US Endangered Species Act, tienen a la especie de berrendo como especie en peligro de extinción y en consecuencia realizan programas para su conservación.

14 Castellanos, Ramón y Sánchez Víctor, "Recuperación del berrendo peninsular”, disponible en: https://studylib.es/doc/5137366/recuperación-del-berrendo-peninsular. Consultado el 4 de octubre del 2019.

15 Abarca, Francisco; Meléndez Cristina; Soria Cynthia; Molina Raul y Corrales Leonardo Colaboración binacional para la conservación del berrendo sonorense, disponible en: http: / / ecologica.jornada.com.mx/2017/07/27//colaboracion-binacional-para-la-conservacion-del-berrendo-sonorense-1440.html, consultada el 22 de enero de 2019.

16 Idem. 
permite a los berrendos cruzar el cerco, ya que no puede saltar como lo harían, por ejemplo, los cimarrones. ${ }^{17}$

Indudablemente, la construcción de un muro en la zona fronteriza afectará el frágil ecosistema de la región, por ejemplo a la población de cactus llamados saguaro, que es un elemento esencial en el desierto, ya que funciona como almacén de agua, alimento y vivienda para aves, como el tecolote pigmeo, que vuela muy bajo, habita en estos gigantes del desierto y los utilizan como trampolín para desplazarse. ${ }^{18}$

Además, existen otros factores que, si bien no necesariamente afectan directamente, si lo hacen en forma indirecta, la construcción de un muro como son la movilización de maquinaria, el uso de tecnologías (helicópteros, luces potentes, por ejemplo, patrullaje, circulación de vehículos, etcétera) que espantan a los animales o afectan sus movimientos naturales. Como es un ecosistema, es decir, son cadenas biológicas que están en equilibrios muy sensibles, cualquier cambio puede afectarlos. ${ }^{19}$

Por otra parte, hay desperdigado en ambos lados de la frontera un complejo de ecosistemas, denominadas "charcas vernales" que aparecen en épocas de lluvias y desaparecen en las sequías y que tienen interrelación entre ellas a pesar de que no hay una distancia entre una y otra. En dichas charcas hay una intensa vida que inclusive se comunica de una o otra. Así, los camarones hada, camarones almeja y camarones renacuajo, son una especie de crustáceos de agua dulce que solo aparecen cuando hay condiciones adecuadas de humedad, lo mismo sucede con cierto tipo de menta. Lógicamente, la construcción de un muro afectará este ecosistema, pues aumentará la fragmentación y hará más difícil la cooperación de los expertos para el estudio de estos sistemas de micro vida que existen en los dos lados de la frontera. ${ }^{20}$

17 Listm, Rurik El bisonte y el muro, disponible en: http://ecologica.jornada.com. $m x / 2017 / 07 / 27 /$ el-bisonte-y-el-muro-2308.html, consultado el 22 de enero de 2019.

18 Ávila Villegas, Sergio, "Impactos de la infraestructura fronteriza en el tecolote pigmeo", disponible en: http://ecologica.jornada.com.mx/2017/07/27/impactos-de-la-infraestructurafronteriza-en-el-tecolote-pigmeo-523.html, consultado el 6 de marzo del 2019.

19 Avila Villegas, Sergio La infraestructura fronteriza y sus efectos en el ambiente común, disponible en: http://ecologica.jornada.com. $m x / 2017 / 07 / 27 /$ la-infraestructura-fronteriza-ysus-efectos-en-el-ambiente-comun-7190.html, consultado el 22 de enero de 2019.

20 García, César, "Ecosistemas únicos: las charcas vernales de la Alta y la Baja California”, disponible en: ecologica.jornada.com.mx/2017/07/27/ecosistemas-unicos-las-charcas-vernales-de- 
También hay que destacar que el mantenimiento del equilibrio natural en esta zona fronteriza requiere cooperación política, armonía entre los dos países; por supuesto, eso no se logra con la construcción de un muro, que es visto como una agresión de Estados Unidos contra su vecino sureño. En ese sentido, el muro en la frontera impacta la relación normal de cooperación binacional. Por ejemplo, la utilización de las aguas del Río Colorado que recorre el territorio de los dos países está regulada por el Tratado de Aguas Internacionales (TAI) firmado por ambos países en el año de 1944. Ahora bien, el TAI es aplicado por la Comisión Internacional de Límites y Aguas (CILA), la cual también define la relación entre ambos países para los ríos Bravo (Grande) y Tijuana. Ahora bien, en el seno de la CILA, los dos Estados fronterizos aprobaron el Acta 319 intitulada "Medidas interinas de cooperación internacional en la cuenca del Río Colorado", que tuvo vigencia hasta el año de 2017. ${ }^{21}$

Ahora bien, esta estructura de cooperación bilateral, que es un canal de negociación con el poderoso vecino norteño, posiblemente con la construcción del muro se dañará, con lo cual los usuarios del agua en el territorio mexicano no tendrán canales de solución de las controversias que son recurrentes en la región.

Del mismo modo hay que mencionar que también un muro impactaría a la Laguna Madre y Delta del Río Bravo en las costas de los Estados de Texas y Tamaulipas y que, a decir de Patricia Moreno Casasola, "constituye una de las reservas más importantes del país y del Golfo de México. Un área de gran importancia económica para la nación y sumamente vulnerable ante los impactos del cambio climático global. La construcción de un muro alterará el funcionamiento de sus dunas y humedales, modificando hábitats y actividades económicas en ambos lados de la frontera". ${ }^{22}$

la-alta-y-la-baja-california-3234.html; consultada el 4 de octubre de 2019. También consultar: www.jardinbotanicosq.com/garden-cafe-1, consultado el 4 de octubre de 2019.

21 Comisión Internacional de Límites y Aguas entre México y los Estados Unidos. Boletín de Prensa, 20 de noviembre del 2012, disponible en: https://studylib.es/doc/7316768/ bolet\%C3\%ADn-de-prensa, consultado el 6 de agosto del 2019.

22 Moreno Casasola, Patricia, "Tamaulipas de cara al mar: un futuro incierto"; disponible en: http: / / ecologica.jornada.com. $\mathrm{mx}_{\mathrm{N}} / 2017 / 07 / 27 /$ tamaulipas-de-cara-al-mar-un-futuro-incierto-5750.html, consultado el 6 de marzo del 2019. 


\section{V. ¿LA GONSTRUCGIÓN DE UN MURO FRONTERIZO ES EJERCICIO LEGAL DE LA SOBERANÍA ESTATAL?}

En sus orígenes, el concepto de soberanía implicaba un poder absoluto que se ejercía sobre un territorio y una población. ${ }^{23}$ Pero, el concepto de soberanía ha evolucionado dramáticamente y, al día de hoy, tiene límites en el orden público internacional. La soberanía clásica que era ejercida ilimitadamente dentro de un territorio concreto ahora ha evolucionado, reconociendo que no se puede ejercer de manera desmedida, pues hay bienes, valores comunes de la humanidad que la limitan. A esos les llamamos áreas mundializadas "que trascienden las soberanías estatales para convertirse en preocupación, interés o responsabilidad mundial, eso significa que no se puede alegar la soberanía interna para no cumplir estándares mundiales creados por el derecho internacional. Nos referimos a: el medio ambiente, los derechos humanos, el derecho humanitario internacional y el derecho penal internacional"; ${ }^{24}$ en otras palabras, áreas jurídicas mundializadas que forman parte de un orden público internacional. En ese sentido, la soberanía estatal, en materia de límites territoriales no es absoluta, sino que está limitada al cumplimiento del orden público internacional.

Ya la Corte Internacional de Justicia (CIJ) se ha pronunciado sobre la construcción de un muro. En este caso, sobre una Opinión Consultiva (OC) solicitada, en 2003 por la Asamblea General de la ONU ${ }^{25}$. La pregunta que le fue sometida a la Corte fue la siguiente:

23 Véase Müller Uhlenbrock, Klaus y BRM, Soberanía y juridificación en las relaciones internacionales, México, UNAM, FES Acatlán, 2010, 273 pp.

24 Becerra Ramírez, Manuel et al., "La soberanía en la era de la globalización”, en Müller Uhlenbrock, Klaus y BRM, op. cit., p. 66.

25 Véase Abi-Saab, Rosemary, "Consecuencias jurídicas de la construcción de un muro en el territorio palestino ocupado”, Revista Internacional de la Cruz Roja, septiembre de 2004; Portilla Gómez, Juan Manuel, "El derecho internacional ante la ocupación de los territorios palestinos y el muro de separación”, Anuario Mexicano de Derecho Internacional, México, vol, VI, 2006, pp. 345-375; Badia Martí, Anna, "La Opinión Consultiva de la Corte Internacional de Justicia sobre las Consecuencias Jurídicas de la Construcción de un Muro en el territorio Palestino Ocupado”, 9 de julio de 2004, disponible en: file:///C:/Users/iij\%20unam/ Downloads /A.Badia(reei9).pdf consultado el 17 de febrero de 2017. 
¿Cuáles son las consecuencias jurídicas que se derivan de la construcción del muro que levanta Israel, la Potencia ocupante, en el territorio palestino ocupado, incluida Jerusalén oriental y sus alrededores, según se describe en el informe del Secretario General, teniendo en cuenta las normas y principios de derecho internacional, ¿en particular el IV Convenio de Ginebra de 1949 y las resoluciones pertinentes del Consejo de Seguridad y de la Asamblea General?

A partir de esta pregunta, en su OC, la CIJ llega a la conclusión que el muro construido por el ejército de Israel en Cisjordania, incluido Jerusalén Oriental y sus alrededores, viola el derecho internacional humanitario y de derechos humanos, y que "Israel también tiene la obligación de poner fin a la violación de sus obligaciones internacionales derivada de la construcción del muro en los Territorios Ocupados palestinos".

Aunque, en principio, la OC es una cuestión de aplicación del derecho humanitario internacional, en su razonamiento también se refiere a la aplicación del derecho internacional de los derechos humanos. En efecto, en el caso del muro construido por los israelís, la CIJ reconoce la aplicación del Pacto Internacional de Derechos Civiles y Políticos y concretamente lo establecido en el párrafo 1 del artículo 2o: "Cada uno de los Estados parte en el presente Pacto se compromete a respetar y a garantizar a todos los individuos que se encuentren en su territorio y estén sujetos a su jurisdicción los derechos reconocidos en el presente Pacto, sin distinción alguna...”.

Por lo tanto, aquí queremos subrayar que la CIJ deja abierta la puerta para la aplicación del derecho internacional de los derechos humanos; es decir, la construcción de un muro no es una cuestión que se encuentre en la facultad soberana de un Estado, el orden público internacional funciona como un elemento de límite a la actuación del Estado.

\section{MARCO JURÍDICO INTERNAGIONAL}

En principio, la construcción de un muro en la frontera es un acto inamistoso entre dos Estados, ya que, es a todas luces evidente que hay rechazo, desprecio al vecino del sur, por cuestiones que son materia de la policía bilateral o multilateral (el tráfico de drogas, la migración del sur al norte, son problemas de carácter multilateral). Básicamente, la vecindad entre los 
Estados fronterizos se regula por normas de convivencia urbana ya que se refiere a la política de buena vecindad, como se expresa en la Carta de San Francisco.

En efecto, en materia de fronteras, ya la Carta de San Francisco en su preámbulo, que es considerado jurídicamente vinculante, establece la obligación de la buena vecindad. ${ }^{26}$ ¿Qué significa ser buen vecino? De acuerdo con la lectura de la Carta de San Francisco significaría que la vecindad trae por consecuencia una serie de obligaciones para los Estados, tales como ser tolerante, esforzarse para mantener la paz y la seguridad internacionales, no agredir, ni utilizar las fuerzas armadas, promover el progreso económico y social; en suma, poner en primer plano la cooperación internacional entre los vecinos. Evidentemente, la política exterior del presidente Donald Trump, viola todas esas obligaciones.

Ahora bien, la construcción del "muro de Trump" afecta a las áreas mundializadas, del medio ambiente y los derechos humanos. Como vimos anteriormente, en cuanto al medio ambiente la construcción de muro afectará a los dos Estados en lo que toca a la flora y fauna silvestre que no reconoce límites estatales, pues son recursos trasnacionales.

Concretamente, el muro y su prolongación afecta la reserva de la Biosfera "El pinacate y Gran desierto de Altar", declarada, en junio de 2013, Patrimonio Natural de la Humanidad por la UNESCO. ${ }^{27}$ Esta zona "se extiende desde Sonora y Baja California Sur hasta Arizona y California en Estados Unidos. Es el hogar de numerosas especies: más de mil de flora y fauna de mamíferos, reptiles, aves, anfibios, peces endémicos. Como vimos, allí viven especies únicas, amenazadas y en peligro de extinción, como el berrendo, el borrego cimarrón, el monstruo de gila y la tortuga de desierto". ${ }^{28}$

Asimismo, el Pinacate forma parte de la red de Reservas del Programa El Hombre y la Biosfera (MAB) de la UNESCO, desde el 25 de octubre de

26 “... a practicar la tolerancia y a convivir en paz como buenos vecinos; a unir nuestras fuerzas para el mantenimiento de la paz y la seguridad internacionales; a asegurar, mediante la aceptación de principios y la adopción de métodos, que no se usará la fuerza armada sino en servicio del interés común, y a emplear un mecanismo internacional para promover el progreso económico y social de todos los pueblos".

27 Disponible en: https://es.wikipedia.org/wiki/Reserva_de_la_biosfera_El_Pinacate_y_ Gran_Desierto_de_Altar, consultada el 12 de febrero de 2017.

28 Restrepo, Iván "El muro: atentado contra la biodiversidad”, La Jornada, México, 30 de enero de 2017. 
1995. Y tiene el nombramiento de los humedales de Agua Dulce como Sitio Ramsar $^{29}$ el 25 de septiembre de 2007, ${ }^{30}$ al mismo tiempo forma parte de la red de Reservas Hermanas del Desierto Sonorense que protegen una amplia y bien conservada franja fronteriza entre Arizona (Estados Unidos) y Sonora (México). ${ }^{31}$

29 El nombre de Ramsar viene de la ciudad iraní del mismo nombre y se refiere a la "Convención Relativa a los Humedales de Importancia Internacional Especialmente como Hábitat de Aves Acuáticas" (Convención Ramsar) celebrada en dicha ciudad el 2 de febrero de 1971. Ahora bien, los Humedales de Importancia Internacional, mejor conocidos como Sitios Ramsar, son áreas que han sido reconocidas internacionalmente al otorgarles una designación de acuerdo con los criterios establecidos por dicha Convención Ramsar, de la que México es parte, desde 1985. Es importante mencionar que los Estados Unidos son parte también de la Convención Ramsar desde 1986, en consecuencia, está vinculado con las obligaciones que impone dicha convención.

Ramsar tiene por objeto proteger, custodiar los humedales, detener su desaparición pues son parte de un ecosistema, lo cual, por si misma ya está provisto de un valor, sin embargo, podemos añadir que tiene una gran importancia económica, cultural, científica y recreacional. Ahora bien, Ramsar construye un sistema de cooperación internacional, pues trabaja estrechamente con organizaciones internacionales asociadas (Birdlife International; International Union for Conservation of Nature (IUCN); International Water Management Institute (IWMI); Wetlands International; WWF International; Wildfowl \& Wetlands Trust (WWT) y redes formadas por tratados como los tratados relativos a la Biodiversidad, la World Heritage Convention (WHC) con fundaciones, con agencias de las Naciones Unidas como el Programa de Naciones Unidas para el medio Ambiente (The United Nations Environment Programme (PNUMA) UNEP). El Programa de Naciones Unidas para el Desarrollo (The United Nations Development Programme (PNUD) UNDP), la UNESCO, etcétera.

30 Agua Dulce Site number: 1813 | Country: Mexico | Administrative region: Sonora Area: 39 ha | Coordinates: $31^{\circ} 55^{\prime} \mathrm{N} 113^{\circ} 01^{\prime} \mathrm{W} \mid$ Designation dates: 02-02-2008 View Site details in RSIS Agua Dulce. 02/02/08; Sonora; 39 ha; $31^{\circ} 55^{\prime} \mathrm{N} 113^{\circ} 01^{\prime} \mathrm{W}$. Located within the Biosphere Reserve Del Picante y Desierto de Altar, which highlights the only riparian ecosystem of the region, Sonoyta river, considered of binational interest and shared between the USA and Mexico. At present, there is a mutual interest in establishing indicators for its management and conservation. Agua Dulce is a $3 \mathrm{~km}$ stretch of the Sonoyta where water comes to the surface, creating conditions of an oasis in a desert. Among the main species found in the site is the Pupfish (Cyprinodon macularius), listed as endangered by the US and as endemic and endangered in Mexico's legal system. There is a considerable presence of the turtle species Kinonsternon sonoriense longifemorale. The resident and migratory bird species that use the Pacific Flyway find in Agua Dulce a habitat of importance for food, shelter, resting and reproduction. Agua Dulce is characteristic for retaining water throughout the year, acting as the main source of water for wildlife in the area, and supporting an excellent biological diversity. Ramsar site no. 1813. Most recent RIS information: 2008.

31 Disponible en: http: / / elpinacate.conanp.gob.mx/ consultada el 12 de febrero de 2017. 
Ahora bien, el artículo 3. 1. del Convenio Ramsar establece la obligación para las partes con relación a los humedales que se encuentren en su territorio, protegidos por la Convención de noticiar sobre el impacto que pudiera tener, por sus acciones: $:^{32}$ Esto es importante, pues la construcción del muro de Trump evidentemente traerá un impacto negativo a los humedales fronterizos y, en principio, la construcción sin notificación es una violación de Ramsar.

Entonces, indudablemente el "Muro trumpiano" viola Ramsar. Además, su construcción va en contra de lo dispuesto en la Convención sobre el Patrimonio de la Humanidad (The World Heritage Convention) de 1972. Esta convención, en esencia, se refiere a la manera cómo el hombre interactúa con la naturaleza y cómo, necesariamente, se debe de preservar un balance entre los dos.

\section{TRATAdos MÉXiCO-Estados Unidos}

Varios tratados celebrados entre México y Estados Unidos conforman el marco normativo que regula las relaciones entre ambos países. Empezando por el tratado de 2 de febrero de 1848, Tratado de Paz, Amistad y Límites o también conocido como Tratado de Guadalupe Hidalgo el tratado de la Mesilla celebrado el 30 de diciembre de 1853. La Convención para la Equitativa Distribución de las Aguas del 21 de mayo de 1906. El Tratado sobre distribución de las Aguas Internacionales celebrado entre México los Estados Unidos de América del 3 de febrero de 1944.

Pero nos detendremos en un tratado que se ha considerado como el que establece un modelo de frontera ecológica es el Convenio para la Coopera-

32 Artículo 3o. del Convenio Ramsar; "Las Partes Contratantes deberán elaborar y aplicar su planificación de forma que favorezca la conservación de los humedales incluidos en la Lista y, en la medida de lo posible, el uso racional de los humedales de su territorio. 2. Cada Parte Contratante tomará las medidas necesarias para informarse lo antes posible acerca de las modificaciones de las condiciones ecológicas de los humedales en su territorio e incluidos en la Lista, y que se hayan producido o puedan producirse como consecuencia del desarrollo tecnológico, de la contaminación o de cualquier otra intervención del hombre. Las informaciones sobre dichas modificaciones se transmitirán sin demora a la organización o al gobierno responsable de las funciones de la Oficina permanente especificado en el artículo 8o.”. 
ción sobre la Protección y la Mejora del Medio Ambiente en la Zona Fronteriza, suscrito en 1983 en la ciudad de la Paz, Baja California, (Convenio de la Paz). ${ }^{33}$ Este tratado es sumamente importante, porque establece el marco jurídico de cooperación en materia ambiental, cuyo objetivo fundamental es preservar un "medio ambiente sano para el bienestar económico y social, a largo plazo, de las generaciones presentes y futuras de cada país, así como de la comunidad internacional”, en él ambos Estados se comprometen a prevenir, reducir o eliminar las fuentes de contaminación de aire, agua y suelo.

Textualmente el Convenio de la Paz establece:

Artículo 1o. Los Estados Unidos Mexicanos y los Estados Unidos de América, en adelante referidos como las Partes, acuerdan cooperar en el campo de la protección ambiental en la zona fronteriza sobre la base de igualdad, reciprocidad y beneficio mutuo. Los objetivos del presente Convenio son establecer las bases para la cooperación entre las Partes en la protección, mejoramiento y conservación del medio ambiente y los problemas que lo afectan, así como acordar las medidas necesarias para prevenir y controlar la contaminación en la zona fronteriza, y proveer el marco para el desarrollo de un sistema de notificación para situaciones de emergencia. Dichos objetivos podrán ser propiciados sin perjuicio de la cooperación que las Partes pudieran acordar llevar a cabo fuera de la zona fronteriza.

Como se ve, el Convenio de la Paz establece las bases para la cooperación en materia de medio ambiente y también prevé la creación de un sistema de notificaciones. Esto significa que, cualquier acción de las partes en la zona protegida por el tratado que afecte el medio ambiente debe ser consultada a la otra. Además, las partes se ven obligadas a "adoptar las medidas apropiadas para prevenir, reducir y eliminar fuentes de contaminación en su territorio respectivo que afecten la zona fronteriza de la otra". ${ }^{34}$

33 Ortiz, Antonio, "Enviromental cooperation in the United Sates-México border, Projet de Recherche Professeur Guy Serge Coté, Politique Environnement, Universidad de Ottawa, abril, 2019.

34 “Artículo 2o. Las partes se comprometen, en la medida de lo posible, a adoptar las medidas apropiadas para prevenir, reducir y eliminar fuentes de contaminación en su territorio respectivo que afecten la zona fronteriza de la otra. Adicionalmente, las partes cooperaran en la solución de problemas ambientales de interés común en la zona fronteriza, de conformidad con las disposiciones de este Convenio". 
Ahora bien, ¿cuál es espacio físico en donde se aplica el Convenio? De acuerdo con este Convenio la "zona fronteriza" es el área situada hasta 100 kilómetros de ambos lados de las líneas divisorias terrestres y marítimas entre las Partes. Precisamente en esa zona "las Partes evaluarán, según sea apropiado, de conformidad con sus respectivas leyes, reglamentos y políticas nacionales, proyectos que puedan tener impactos significativos en el medio ambiente de la zona fronteriza, para que se puedan considerar medidas apropiadas para evitar o mitigar efectos ambientales adversos". ${ }^{35}$ Dicho en otras palabras, la soberanía de los dos Estados, de acuerdo con el derecho internacional está limitada, los Estados Unidos no puede actuar en forma unilateral como la hace con la expedición de la Orden Ejecutiva.

En esta misma dirección y en el campo del llamado soft law, podemos citar la Resolución 3129 (XXVII) "Cooperación en el campo del medio ambiente en materia de recursos naturales compartidos por dos o más Estados", de la Asamblea General de Naciones Unidas, de fecha 13 de diciembre de 1973 se establece la obligación de cooperar y además de informarse y consultarse mutuamente. ${ }^{36}$ Por lo cual, nuevamente estamos viendo que el Presidente de los Estados Unidos no puede actuar de manera unilateral, es necesario cooperar, informar y consultar a México.

\section{LOS REGURSOS TRANSFRONTERIZOS}

En el siglo pasado es perceptible un progreso importante en el derecho internacional del medio ambiente que ha roto el paradigma de la supremacía del Estado, en todos sus aspectos internos. Ese desarrollo ha sido funda-

35 Artículo 7 de la Convención de la Paz.

36 La Resolución establece: "1. Estima que es necesario asegurar una cooperación eficaz entre los países mediante el establecimiento de normas internacionales adecuadas para la conservación y explotación armoniosa de los recursos naturales comunes a dos o más Estados en el marco de las relaciones normales existentes entre ellos.

“2. Estima también que la cooperación entre países que comparten dichos recursos naturales y están interesados en su explotación debe desarrollarse sobre la base de un sistema de información y de consultas previas en el marco de las relaciones normales que existen entre ellos.

“3. Pide al Consejo de Administración del Programa de las Naciones Unidas para el Medio Ambiente que... 
mentalmente del derecho consuetudinario, más no de las normas convencionales. En este fenómeno tiene que ver el avance tecnológico que se ha alcanzado con las revoluciones industriales y que han puesto en jaque al medio ambiente global; es decir, pone en peligro al mismo ser humano en la tierra. Sin embargo, las grandes potencias industriales, como los Estados Unidos que, es causante en gran medida del deterioro del medio ambiente a nivel mundial, no han tenido la voluntad de crear normas convencionales, que establezcan mecanismos para que los Estados se limiten en su acción contaminante. Precisamente, los Estados Unidos ve a las normas del medio ambiente como una limitante a su actividad y supremacía industrial, lo mismo pasa con China. Pero, en forma paradójica, Estados Unidos ha tenido que recurrir al derecho internacional para defenderse de la acción contaminante de su vecino, Canadá, como lo veremos con el famoso caso Smelter.

Como afirmamos, en el derecho internacional contemporáneo, la soberanía estatal ya no significa una facultad omnímoda de los poderes del Estado para hacer y deshacer dentro del territorio. Las limitaciones las establece el mismo orden jurídico en toda su extensión, tanto el convencional como el consuetudinario. Precisamente, el derecho consuetudinario en el caso del Muro que planea Trump es muy importante, pues la gran potencia mundial, en lo que se refiere al derecho convencional es muy reticente a aceptarlo. En efecto, los Estados Unidos no es parte de los grandes tratados como la Convención de Viena sobre Derechos de los Tratados de 1969, la Convención sobre Derecho del Mar de Montego Bay de 1982, la Convención sobre Biodiversidad, de Río de Janeiro de 1992, aún más de tratados regionales, como la Convención Americana de Derechos Humanos de 1969 y muchos otros más. Es por eso, que el derecho consuetudinario, es muy importante para relacionarse con ese país.

Así, podemos encontrar una serie de normas consuetudinarias y principios que reconocen las limitaciones del Estado. Por ejemplo, ya desde principios del siglo pasado el jurista alemán, nacionalizado inglés Lassa Oppenheim, escribía en su Tratado sobre derecho internacional: "A state, in spite of its territorial supremacy is not allowed to alter the natural conditions of its own territory to the disantages of the natural conditions of territory of neighbouring States". ${ }^{37}$ En efecto, Oppenheim, visualizaba una transfor-

37 Oppenheim, Lassa, International Law, UK, p. 220, citado por Chinthaka Mendis, "Sovereignty vs. trans-boundary environmental harm. The evolving international law obligations 
mación trascendente del derecho internacional westphaliano en donde el territorio estaba sujeto sólo a la soberanía interna y no podía ser de otra manera, pues se trataba de crear los Estados europeos.

Actualmente es una obligación del derecho consuetudinario internacional no actuar de tal manera que se causen daños a los derechos de otros Estados. Esta obligación se ha reflejado en la jurisprudencia internacional, en varios casos que ahora son un referente esencial del derecho internacional del medio ambiente; como son los casos de Islas de Palmas, Trail Smelter Arbitration (USA vs. Canada, 1938); Corfu Chanel case (UK vs. Albania), 1949); Lake Lanoux Arbitration (Spain vs. France 1957); Nuclear test case (New Zealand vs. France); Case concerning the Gabcikovo-Nagymaros project (Hungary vs. Slovakia); Pulps Mills case Regarding the River Uruguay (Argentina Uruguay). ${ }^{38}$

En principio, en el Caso Isla de Palmas (EEUU c/Países Bajos) Sentencia arbitral se incluía el concepto de la soberanía territorial, pero también incluía la obligación para los Estados de proteger los derechos de otros Estados dentro de su territorio. ${ }^{39}$

Otro caso muy importante para nuestro tema es el caso arbitral Trail Smelter, Estados Unidos vs Canadá, de 1949. En este caso, Estados Unidos alegó que la empresa Canadian Smelter Factory, situada muy cerca de la frontera con Estados Unidos, en territorio de Canadá estaba causando contaminación y daños a los árboles, cultivos y tierras en el territorio de los Estados Unidos y el tribunal decidió que:

...under principles of international law, as well as the law, as well as the law of the US, no states has the right to use or permite the use of territory in such manner as to cause injury by fumes in or to the territory of another or the properties or persons therein, when the case is of serious consecuences and the injury is established by clear and convincing evidence. ${ }^{40}$

and the Sethusamuduram Ship Chanel Proyect", United Nations/ Nippon Foundation Felow, 2006 .

38 No está por demás decirlo que, dada la importancia del tema ambiental, en 1993 la Corte Internacional de Justicia instaló una Cámara para el derecho ambiental.

39 Malin, Per, State Responsability in Relation to Transboundary Enviromental Damage, Faculty of Law University of Lund, Suecia, primavera de 2007.

4035 AJIL, 1941, pp. 182 and 35 AJIL, 1941, p. 648. 
El caso es muy importante para nosotros por dos razones; en principio, porque los Estados Unidos, fue el demandante y además fue exitoso en su demanda. Lo que significa que el país está consciente de sus obligaciones internacionales, no puede demandar al vecino del norte y omitir sus deberes con el vecino del sur. Después porque es un caso seminal en materia de medio ambiente, en donde, por primera vez un tribunal internacional decidió que ningún Estado puede causar daños desde su territorio al territorio del Estado vecino.

Otro caso, trascendente para nuestro tema es el caso Corfu Chanel, en donde el Reino Unido de la Gran Bretaña demandó a Albania ante la Corte Internacional de Justicia porque se causaron daños a dos barcos, el Saumarez and Volage, por la explosión de minas, colocadas en el paso internacional, dentro de aguas de Albania, en un área al sur oeste de la Bahua de Saranda. Aparte de los daños a los barcos, la explosión de las minas causó muertes y heridos de personas que navegaban en los dos barcos militares. La Corte, decidió:

The obligations incumbent upon the Albanian authorities consisted in notifying, for the benefit of shipping in general, the existence of a minefield in Albanian territorial waters and in warning the approaching British warships of the imminent danger to which the minefield exposed them. Such obligations are based, not on the Hague Convention of 1907, No. VIII, which is applicable in time of war, but on certain general and well-recognized principles, namely : elementary considerations of humanity, even more exacting in peace than in war ; the principle of the freedom of maritime communication; and every State's obligation not to allow knowingly its territory to be used for acts contrary to the rights of other States (las cursivas son añadidas). ${ }^{41}$

De la lectura de este pasaje de la sentencia deriva un principio bien reconocido que establece que los Estados tienen la obligación de no permitir, a sabiendas, que su territorio sea usado para actos contrarios a los derechos de otros Estados. Lo trascendente aquí, es que ya, para la fecha de la sen-

${ }^{41}$ Cour Internationale de Justice, "Recueil des arrêts, avis consultatifs et ordonnances. Affaire du Detroit de Corfou (fond)”, Arret du 9 avril 1949 International Court of Justice, Reports of judgments, advisory opinions and orders the corfu channel case (merits) judgment of april 9th, 1949, p. 22, disponible en: https://www.icj-cij.org/files/case-related/1/00119490409-JUD-01-O0-EN.pdf, consultado el 12 de marzo del 2019. 
tencia, el derecho de una soberanía total, dentro de las fronteras, estaba cuestionado.

Por otra parte, la jurisprudencia internacional tiene otros casos trascendentes aplicables al caso que nos ocupa. Por ejemplo, el caso arbitral de Lake Lanoux Arbitration (Spain vs France 1957) trató sobre el uso de las aguas del lago Lanoux en los Pirineos. El litigio surgió porque el Gobierno Francés propuso la realización de ciertos trabajos para la utilización de las aguas y el gobierno español temía que dichos trabajos pudieran afectar sus derechos e intereses en violación con el Tratado de Bayona del 26 de mayo del año 1866 y el Acta Adicional del mismo año. Entre otras expresiones el tribunal arbitral expresó:

11. [...] To admit that jurisdiction in a certain field can no longer be exercised except on the condition of, or by way of, an agreement between two States, is to place an essential restriction on the sovereignty of a State, and such restriction could only be admitted if there were clear and convincing evidence... ${ }^{42}$

Es evidente que la soberanía interna de los Estados tiene limitaciones en los derechos de los demás Estados; cualquier afectación a la soberanía de otro Estado requiere un acuerdo de voluntades.

En la década de los años setenta, concretamente en 1973, Australia y Nueva Zelanda, cada Estado por su parte, demandaron a Francia ante la Corte Internacional de Justicia por las pruebas nucleares que dicha potencia nuclear detonaba y por supuesto, afectaba el medio ambiente de la región y particularmente a los dos Estados demandantes. Esta sentencia tiene importancia para nosotros, pues la Corte puntualiza el enorme valor que tiene en el derecho internacional contemporáneo el principio de buena fe.

One of the basic principles governing the creation and performance of legal obligations, whatever their source, is the principle of good faith. Trust and confidence are inherent in international Cooperation, in particular in an age when this Cooperation in many fields is becoming increasingly essential. Just as the very rule of

42 Lake Lanoux Arbitration (France vs. Spain) (1957) 12 R.I.A.A. 281; 24 I.L.R. 101 Arbitral Tribunal.1 November 16, 1957, disponible en: https: / /www.informea.org/sites/default / files/court-decisions/COU-143747E.pdf, consultado el 12 de marzo del año 2019. 
pacta sunt servanda in the law of treaties is based on good faith, so also is the binding character of an international obligation assumed by unilateral declaration.

Es decir, en el sistema de relaciones internacionales un principio fundamental es el de pacta sunt servanda que no se está cumpliendo en la relación México-Estados Unidos. Como vimos, estos dos países tienen un entramado de tratados bilaterales de medio ambiente que tienen la obligación de cumplir.

Por otra parte, en 1994 la Asamblea General de la ONU solicitó a la Corte Internacional de Justicia (CIJ) una opinión consultiva sobre ¿si la amenaza o uso de las bombas atómicas, en cualquier circunstancia, está permitido por el derecho internacional? En su Opinión la Corte llegó a una conclusión insólita, desde la perspectiva del derecho internacional, y fue que se declaró non liquet, cuando desde nuestra óptica el sistema de derecho internacional es hermético, y todos los casos que se presenten tienen un marco jurídico. Pero, aquí nos interesa traer a colación el reconocimiento que hace la misma Corte al medio ambiente, cuando expresa que:

29. The Court recognizes that the environment is under daily threat and that the use of nuclear weapons could constitute a catastrophe for the environment. The Court also recognizes that the environment is not an abstraction but represents the living space, the quality of life and the very health of human beings, including generations unborn. The existence of the general obligation of States to ensure that activities within their jurisdiction and control respect the environment of other States or of areas beyond national control is now part of the corpus of international law relating to the environment. ${ }^{43}$

De la lectura de este párrafo se puede afirmar que, no existe más en derecho internacional contemporáneo una soberanía ilimitada sobre el territorio de un Estado y se tocan los siguientes puntos:

- Se reconoce que el medio ambiente se encuentra en una amenaza constante;

43 Corte Internacional de Justicia, Legality of the Threat or Use of Nuclear Weapons, Advisory Opinion of 8 July 1996; https: / /www.icj-cij.org/en/case/95/advisory-opinions. 
- también se reconoce que el medio ambiente no es una abstracción pues representa el espacio vivo, la calidad de vida y la salud del ser humano, incluyendo a las generaciones futuras;

- por último, se reconoce la obligación de respetar al medio ambiente de otros Estados, con las actividades dentro de su territorio.

\section{EL PRINCIPIO DE NO PERJUDICAR AL MEDIO AMBIENTE DE OTRO ESTADO, CON ACTIVIDADES DENTRO DE SU TERRITORIO}

En el ejercicio de analizar la legalidad de la construcción del muro en la frontera de Estados Unidos-México, es importante mencionar un principio toral para el tema y es precisamente el de "no perjudicar al medio ambiente de otros Estados con actividades dentro de su territorio". Este principio lo encontramos en el derecho convencional multilateral, y también tiene una naturaleza consuetudinaria. Por eso, no es posible que los Estados Unidos aleguen que no son parte de muchos tratados internacionales multilaterales, para no verse obligado por este principio de carácter consuetudinario. Como vimos, con el caso Smelter Arbitration no sería tampoco posible que alegue que es un objetor persistente.

Estas mismas obligaciones se pueden ver en el derecho convencional, en donde, por ejemplo, en la III Conferencia Sobre Derecho del Mar, de Montego Bay, se refiere a esas limitaciones que tienen los Estados de explotar sus recursos naturales, con limitaciones, como se puede ver en sus artículos 193 y $194-2 .^{44}$

44 Artículo 193 Derecho soberano de los Estados de explotar sus recursos naturales Los Estados tienen el derecho soberano de explotar sus recursos naturales con arreglo a su política en materia de medio ambiente y de conformidad con su obligación de proteger y preservar el medio marino.

Artículo 194 Medidas para prevenir, reducir y controlar la contaminación del medio marino.

2. Los Estados tomarán todas las medidas necesarias para garantizar que las actividades bajo su jurisdicción o control se realicen de forma tal que no causen perjuicios por contaminación a otros Estados y su medio ambiente, y que la contaminación causada por incidentes o actividades bajo su jurisdicción o control no se extienda más allá de las zonas donde ejercen derechos de soberanía de conformidad con esta Convención. 
Lo mismo en el denominado soft law, la Declaración de Estocolmo, en su principio 21 establece:

De conformidad con la Carta de las Naciones Unidas y con los principios del derecho internacional, los Estados tienen el derecho soberano de explotar sus propios recursos en aplicación de su propia política ambiental, y la obligación de asegurarse de que las actividades que se lleven a cabo dentro de su jurisdicción o bajo su control no perjudiquen al medio ambiente de otros Estados o de zonas situadas fuera de toda jurisdicción nacional. ${ }^{45}$

Asimismo, en la Declaración de Río sobre el Medio Ambiente y el Desarrollo de 1992, se toma esa misma dirección, en su principio 2 en donde se estableció:

De conformidad con la Carta de las Naciones Unidas y los principios del derecho internacional, los Estados tienen el derecho soberano de aprovechar sus propios recursos según sus propias políticas ambientales y de desarrollo, y la responsabilidad de velar porque las actividades realizadas dentro de su jurisdicción o bajo su control no causen daños al medio ambiente de otros Estados o de zonas que estén fuera de los límites de la jurisdicción nacional (las cursivas son añadidas).

Esta es una obligación de carácter erga omnes, según se desprende de la interpretación que se hace al artículo 48 del Proyecto sobre responsabilidad internacional preparado por la Comisión de Derecho Internacional que habla de que

... todo Estado que no sea un Estado lesionado tendrá derecho a invocar la responsabilidad de otro Estado...si la obligación violada existe con relación a un grupo de Estados del que el Estado invocante forma parte y ha sido establecida para la protección de un interés colectivo del grupo; o la obligación violada existe con relación a la comunidad internacional en su conjunto.

Más adelante, en el artículo 3o. Del Convenio sobre la Diversidad Biológica CDB del año de 1992, se establece:

45 Declaración de Estocolmo Sobre el Medio Ambiente Humano Adopción: Conferencia de las Naciones Unidas sobre el Medio Ambiente Humano, 16 de junio de 1972. 
Principio de conformidad con la Carta de las Naciones Unidas y con los principios del derecho internacional, los Estados tienen el derecho soberano de explotar sus propios recursos en aplicación de su propia política ambiental y la obligación de asegurar que las actividades que se lleven a cabo dentro de su jurisdicción o bajo su control no perjudiquen al medio de otros Estados o de zonas situadas fuera de toda jurisdicción nacional" (las cursivas son nuestras).

Hay que insistir que si bien los Estados Unidos, no es parte de la CDB, sin embargo, este principio es parte del derecho consuetudinario internacional y claramente establece una limitación a la soberanía territorial de los Estados.

\section{Conclusiones}

Sobre todo, a partir de la segunda mitad del siglo XX, el derecho internacional contemporáneo ha evolucionado hacia la creación de un orden público internacional compuesto de normas de derechos humanos, medio ambiente y derecho penal internacional. Ese orden público hace que no sea posible alegar la existencia de la soberanía interna de los Estados, para realizar actos que dañen los derechos de los vecinos.

El ecosistema existente en la frontera entre Estados Unidos y México es muy rico en variedad de plantas y animales, sin embargo, se encuentra en una situación de gran fragilidad, justamente por la acción del hombre a través de los años. Precisamente, la casi extinción del bisonte que dominaba las grandes llanuras del territorio que ahora es de los Estados Unidos, es un ejemplo de cómo la acción descontrolada del hombre puede casi desaparecer una especie. Lo malo, es que hay otras variedades que pueden desaparecer con una acción tan violenta como la construcción de un muro.

El muro construido en la frontera México-Estados Unidos, así como, los planes de su prolongación por parte de la presidencia de los Estados Unidos, son violatorios de las normas jurídicas internacionales, convencionales, tanto bilaterales como multilaterales, así como, de las normas consuetudinarias; e inclusive de las normas políticas, de la buena vecindad y de las 
normas morales, que no se analizaron en este trabajo, pero son evidentes, al colocar muros que dañan irreparablemente el medio ambiente.

Es claro que, los Estados Unidos violan la normatividad internacional en materia de medio ambiente con la construcción de un muro en la frontera con México. Por lo tanto, incurre en responsabilidad internacional por la comisión de un acto ilícito, como lo señala el artículo 1o. y 2o. del Proyecto de artículos sobre la responsabilidad internacional, que fue aprobada por la Comisión de Derecho Internacional de la ONU, ${ }^{46}$ en 2001 y en consecuencia Estados Unidos tiene las siguientes obligaciones:

- Debe de cumplir con la obligación violada (artículo 29).

- Debe de ponerle fin a la violación, si continúa y ofrecer seguridades y garantías adecuadas de no repetición, si las circunstancias lo exigen (artículo 30).

- Reparar íntegramente el perjuicio causado por el hecho internacionalmente ilícito. En el entendido de que el perjuicio comprende todo daño, tanto material como moral, causado por el hecho internacionalmente ilícito (artículo 31).

\section{BiBLIOGRAFÍA}

ABARCA, Francisco et al., "Colaboración binacional para la conservación del berrendo sonorense", disponible en: http://ecologica.jornada.com. $\mathrm{mx}_{\mathrm{x}} / 2017$ /07 / 27 / colaboracion-binacional-para-la-conservacion-del-berrendo-sonorense-1440.html.

ABI-SAAB, Rosemary, "Consecuencias jurídicas de la construcción de un muro en el territorio palestino ocupado", Revista Internacional de la Cruz Roja, septiembre de 2004.

Anglés HernándeZ, Marisol, "Los cursos de agua compartidos entre México y los Estados Unidos de América y la variable medio ambiental. Una aproximación", Anuario Mexicano de Derecho Internacional, México, vol. VI, 2006.

46 Responsabilidad del Estado por hechos internacionalmente ilícitos (AG/56/83). 
Esta revista forma parte del acervo de la Biblioteca Jurídica Virtual del Instituto de Investigaciones Jurídicas de la UNAM

Ávila Villegas, Sergio, "Impactos de la infraestructura fronteriza en el tecolote pigmeo”, disponible en: ecologica.jornada.com. $m_{x} / 2017 / 07 / 27 /$ impactos-de-la-infraestructura. http://ecologica.jornada. com.mx/2017/07/27/impactos-de-la-infraestructura-fronteriza-en-el-tecolote-pigmeo-523.html.

BADIA MARTí, Anna, "La opinión consultiva de la Corte Internacional de Justicia sobre las consecuencias jurídicas de la construcción de un muro en el territorio Palestino Ocupado”, 9 de julio de 2004, disponible en: file:/ / / C: / Users/iij\%20unam/Downloads/A.Badia(reei9).pdf.

Becerra Ramírez, Manuel, Povedano Amezola, Adriana, Téllez Carvajal, Evelyn "la soberanía en la era de la globalización”, en MÜLLER UHLENBROCK, Klaus y BRM, Soberanía y juridificación en las relaciones internacionales, México, UNAM, FES Acatlán, 2010.

CASTEllanos, Ramón y SÁNCHEZ Víctor, "Recuperación del berrendo peninsular", disponible en: ttps://studylib.es/doc/5137366/recuperacióndel-berrendo-peninsular.

GARCÍA, César, "Ecosistemas únicos: las charcas vernales de la Alta y la Baja California”, disponible en: ecologica.jornada.com.mx/2017/07/27/ecosistemas-unicos-las-charcas-vernales-de-la-alta-y-la-baja-california-3234.html.

HeRnándeZ, Héctor, M., La vida en los desiertos mexicanos, México, Fondo de Cultura Económica, México, 2006.

ORTEGA,Yemeli, “Trump wall threatens Mexico’s animals without borders”, April 9, 2017, disponible en: https: / / phys.org/news /2017-04-trump-wallthreatens-mexico-animals. $h t m l \# j C p$.

LASKY, Jesse R, Jetz Walter y Keitt, Timothy H. , "Conservation biogeography of the US-Mexico border: a transcontinental risk assessment of barriers to animal dispersal", Diversity and Distributions. A Journal of Conservation Biogeograpy, 3 de mayo de 2011, disponible en: https: / doi. org/10.1111/j.1472-4642.2011.00765.x.

LISTM, Rurik, "El bisonte y el muro", disponible en: http://ecologica.jornada.com.mx/2017/07/27/el-bisonte-y-el-muro-2308.html.

Malin, Per, State Responsability in Relation to Transboundary Enviromental Damage; Faculty of Law University of Lund, Suecia, primavera de 2007

Moreno Casasola, Patricia, "Tamaulipas de cara al mar: un futuro incierto"; disponible en: http://ecologica.jornada.com.mx/2017/07/27/ tamaulipas-de-cara-al-mar-un-futuro-incierto-5750.html. 
Esta revista forma parte del acervo de la Biblioteca Jurídica Virtual del Instituto de Investigaciones Jurídicas de la UNAM

MüLler UHLENBROCK, Klaus y BRM, Soberanía y juridificación en las relaciones internacionales, México, UNAM, FES Acatlán, 2010.

PORTILla GÓMEZ, Juan Manuel, “El derecho internacional ante la ocupación de los territorios palestinos y el muro de separación”, Anuario Mexicano de Derecho Internacional, México, vol. VI, 2006. 